Communications in Physics, Vol. 20, No. 3 (2010), pp. 241-247

\title{
MAGNETOPHONON RESONANCE IN QUANTUM WELLS WITH PARABOLIC POTENTIAL
}

\author{
BUI DINH HOI \\ National University of Civil Engineering \\ VO THANH LAM \\ Sai Gon University \\ TRAN CONG PHONG \\ Hue University of Education
}

\begin{abstract}
The linear dc magnetoconductivity in the $(x, y)$ plane of a parabolic quantum well, with a magnetic field $\vec{B}=B \vec{e}_{z}$ applied, is evaluated for electron - optical phonon interaction. For nonpolar optical and polar optical phonons, the magneto-conductivity oscillates as a function of the magnetic field with resonances occurring when $P \omega_{c}=\omega_{0}$, where $\omega_{c}$ and $\omega_{0}$ are cyclotron frequency and optical phonon frequency, respectively, and where $P$ is an integer. The analytic results are numerically evaluated to show explicitly the dependence of magneto-conductivity on the magnetic field, the confinement frequency in $z$ direction, and the temperature of the system.
\end{abstract}

\section{INTRODUCTION}

Over the past few decades, many studies of magnetophonon resonance (MPR) effects in bulk semiconductor systems [1-5], quasi-two-dimensional (Q2D) quantum well systems [6-12], and quasi-one-dimensional (Q1D) quantum wire systems [13-15] have been reported in considerable details from both experimental and theoretical points of view. The MPR effects arise from an electron resonant scattering due to absorption and emission of phonons when energy separation between two Landau levels equals the longitudinal-optical (LO) phonon energy. Warmenbol et al. [9] studied the hot electrons in two-dimensional system (formed in a single heterojunction) theoretically on the basis of the momentum-balance equation. Mori et al. [10] also studied the same system using Kubo formula and the FangHoward trial function. For Q2D quantum well (superlattice) structures, Vasilopoulos et al. $[6,7]$ studied the hot-electron MPR and the magneto-impurity resonance for various scattering mechanisms, including optical phonon and impurity scattering, utilizing the method adopted by Barker [11]. For optical phonons in the quantum well with square confining potential, Vasilopoulos [6] showed that the MPR occurring when $\omega_{L}=P \omega_{c}$, where $P$ is an integer and $\omega_{L}$ and $\omega_{c}$ are the optical phonon frequency and cyclotron frequency, respectively.

The MPR effects are powerful spectroscopic tools for investigating the transport properties of semiconductor, such as the carrier relaxation mechanism, the damping of oscillation due to electron-phonon interaction, the measurement of the effective mass, and the determination of the energy difference between two adjacent electric subbands. So the 
purpose of present work is to apply the magnetoconductivity formula in [17] to evaluate the conductivity tensor component $\sigma_{x x}$ in a quantum well with parabolic confining potential in the presence of a magnetic field in $z$ direction (the confining direction) $\vec{B}=B \overrightarrow{e_{z}}$. We consider only the scattering of electrons by longitudinal-optical (LO) phonons (nonpolar and polar optical phonons) in the deformation potential model. The results show an explicit oscillatory behavior of the conductivity as a function of the magnetic field. For optical phonons the resonance condition is $\omega_{L}=P \omega_{c}$. Since most of the results involve the Landau level widths, we evaluate the relevant inverse scattering rates as well. These rates then are used to extract the level widths. The dependence of the results on the magnetic field, the confinement frequency, and the temperature of sample is shown explicitly. The present paper is organized as follows: In Sec. II we present briefly the basic formulas for the calculation. In the next section, we briefly describe the simple model of a quantum well and evaluate analytically the dc magnetoconductivity for two scattering mechanism. Numerical results and discussion are given in Sec. IV. Finally, remarks and conclusions are shown briefly in Sec. V.

\section{BASIC FORMULAS}

We consider a many body system with a Hamiltonian given by

$$
H=H^{0}+\lambda V-A F(t)
$$

$H^{0}$ is the largest part of $H$ which can be diagonalized, $\lambda V$ is a binary type interaction, assumed nondiagonal, and $-A F(t)$ is the external field Hamiltonian with $A$ being an operator and $F(t)$ a generalized force.

In [16] the Hamiltonian given by Eq. (1) was inserted into von Neumann's equation for the density operator $\rho$ which was split into a diagonal $\left(\rho_{d}\right)$ and a nondiagonal $\left(\rho_{n d}\right)$ part. Then by means of projection operators and for linear response, two inhomogeneous master equations (diagonal and nondiagonal) [16] as well as a diagonal [16] and a nondiagonal quantum Boltzmann's equation were obtained. They are valid in van Hove limit, $\lambda \rightarrow$ $0,1 / \tau \rightarrow \infty, \lambda^{2} t=$ finite, where $\tau$ is the time for a transition between two eigenstates of $H^{0}$ to take place, $\tau \simeq \hbar / \delta \varepsilon$ (this equivalent to the first Born approximation).

For Landau states, the diagonal dc conductivity tensor is given by $[6,7,17]$

$$
\sigma_{\mu \nu}^{d}(0)=\frac{\beta e^{2}}{\Omega} \sum_{\xi, \xi^{\prime}}<n_{\xi}>_{e q}\left(1-<n_{\xi^{\prime}}>_{e q}\right) w_{\xi \xi^{\prime}}\left(R_{\nu \xi}-R_{\nu \xi^{\prime}}\right) R_{\mu \xi^{\prime}}
$$

where $\Omega$ is the normalization volume of sample; $e$ the charge of the carriers; $\sum_{i}\left(\vec{r}_{i}-<\right.$ $\left.\vec{r}_{i}>_{e q}\right)=\sum_{i} \vec{\alpha}_{i} ; \vec{r}_{i}$ is the position of carrier $i$, and $\left\langle\vec{r}_{i}\right\rangle_{e q}$ is its equilibrium position prior to the switching on of the field; $R_{\mu \xi} \equiv \alpha_{\mu \xi}=<\xi|\mu| \xi>; \mu, \nu=x, y, z ; \beta=1 /\left(k_{B} T\right)$ with $k_{B}$ being Boltzmann's constant, and $T$ the temperature; $\left\langle n_{\xi}\right\rangle_{e q}$ and $\left\langle n_{\xi^{\prime}}\right\rangle_{e q}$ are the average occupancies of the eigenstates $|\xi\rangle$ and $\left|\xi^{\prime}\right\rangle$, respectively, given by Fermi-Dirac distribution functions; $w_{\xi \xi^{\prime}}$ is the binary transition rate (transition probability) between two corresponding eigenstates $|\xi\rangle$ and $\left|\xi^{\prime}\right\rangle$, given by "golden rule".

Formula (2), first derived by Argyres and Roth [18], for $\mu=\nu=x$, is valid for both elastic $\left(<n_{\xi}>_{e q}=<n_{\xi^{\prime}}>_{e q}\right)$ and inelastic $\left(<n_{\xi}>_{e q} \neq<n_{\xi^{\prime}}>_{e q}\right)$ scattering. 
The above formula is fairly general and not tied to a $\vec{k}$ space description. In the case of electron-phonon interaction (we assume that the phonons remain at equilibrium), $H^{0}$, in Eq. (1), contains an electron part and a phonon part; $\lambda V$ is the usual electron-phonon interaction. In this case the transition probabilities $w_{\xi \xi^{\prime}}$ are given by $[6,7,17]$

$$
w_{\xi \xi^{\prime}}=\sum_{\vec{q}}\left[Q\left(\xi, \vec{q} \rightarrow \xi^{\prime}\right)<N_{\vec{q}}>_{e q}+Q\left(\xi \rightarrow \xi^{\prime}, \vec{q}\right)\left(1+<N_{\vec{q}}>_{e q}\right)\right]
$$

where

$$
\begin{aligned}
& Q\left(\xi, \vec{q} \rightarrow \xi^{\prime}\right)=\frac{2 \pi}{\hbar}|C(\vec{q})|^{2}\left|<\xi^{\prime}\right| e^{i \vec{q} r}|\xi>|^{2} \delta\left(\varepsilon_{\xi}-\varepsilon_{\xi^{\prime}}+\hbar \omega_{\vec{q}}\right), \\
& Q\left(\xi \rightarrow \xi^{\prime}, \vec{q}\right)=\frac{2 \pi}{\hbar}|C(\vec{q})|^{2}\left|<\xi^{\prime}\right| e^{-i \vec{q} \vec{r}}|\xi>|^{2} \delta\left(\varepsilon_{\xi}-\varepsilon_{\xi^{\prime}}-\hbar \omega_{\vec{q}}\right) .
\end{aligned}
$$

The first and second term of Eq. (3) stand for the absorption and emission of a phonon of wave vector $\vec{q}$ with energy $\hbar \omega_{\vec{q}}$, respectively. The notation $\left(\xi, \vec{q} \rightarrow \xi^{\prime}\right)$ in the first term means that an electron at state $|\xi\rangle$ absorbs a phonon of wave vector $\vec{q}$ to change its state to $\left|\xi^{\prime}\right\rangle$, and in the second term, the notation $\left(\xi \rightarrow \xi^{\prime}, \vec{q}\right)$ expresses the transition of an electron from state $|\xi\rangle$ to state $\left|\xi^{\prime}\right\rangle$ by emitting a phonon of wave vector $\vec{q} .<N_{\vec{q}}>_{e q}$ is the average number of phonons, given by Bose-Einstein distribution function. We consider only LO-phonons as treated by the deformation potential model, also we assume that optical phonons are not dispersion, i. e., $\hbar \omega_{\vec{q}} \simeq$ const, this means that $\left\langle N_{\vec{q}}\right\rangle_{e q}$ does not depend on wave vector $\vec{q}$ of phonons, so we can set $\left\langle N_{\vec{q}}\right\rangle_{e q}=N_{0}$ (depends only on the temperature of system) for short writing.

\section{APPLICATIONS FOR MODEL OF A PARABOLIC QUANTUM WELL}

We consider the transport of an electron gas in a quantum well structure, in which a one-dimensional electron gas is confined in a heterostructure by a parabolic potential $U(z)$ along the $z$ direction, and electron motions are free along two directions (the $(x, y)$ plane). A static magnetic field $\vec{B}$ is applied in the $z$ direction. Then, the one-electron Hamiltonian $\left(H^{0}=\sum h^{0}\right)$, its normalized eigenfunctions $(|\xi\rangle)$, and the eigenvalues $\left(\varepsilon_{\xi}\right)$ in the Landau gauge for the vector potential $\vec{A}=(0, B x, 0)$ are, respectively, given by [8]

$$
\begin{aligned}
h^{0} & =(\vec{p}+e \vec{A})^{2} / 2 m^{\star}+U(z), \\
|\xi\rangle \equiv\left|N, n, k_{y}\right\rangle & =\frac{1}{\sqrt{L_{y}}} \exp \left(i k_{y} y\right) \phi_{N}\left(x-x_{0}\right) \psi_{n}(z), \\
\varepsilon_{\xi} \equiv \varepsilon_{N, n, k_{y}} & =\left(N+\frac{1}{2}\right) \hbar \omega_{c}+\varepsilon_{n} ; N=0,1,2, \ldots,
\end{aligned}
$$

where $m^{\star}$ is the effective mass of a conduction electron with electron charge $-e(e>0)$, $\vec{p}$ is the momentum operator of a conduction electron, $N$ is the Landau level index and $n$ denotes level quantization in $z$ direction, and $\omega_{c}=e B / m^{\star}$ is the cyclotron frequency. Also, $\phi_{N}$ represents harmonic oscillator wave functions, centered at $x_{0}=-\hbar k_{y} / m^{\star} \omega_{c}$. Here $k_{y}$ is the wave vector in the $y$ direction and $L_{y}$ is the $y$-directional normalization length. The radius of the orbit in the $(x, y)$ plane is $l_{B}=\left(\hbar / m^{\star} \omega_{c}\right)^{1 / 2}$. For a parabolic 
well given by $U(z)=m^{\star} \omega_{z} z^{2} / 2$ with the characteristic frequency $\omega_{z}$ of the confinement potential, the one-electron normalized eigenfunctions and the corresponding eigenvalues in the conduction band are, respectively, given by [8]

$$
\begin{aligned}
\psi_{n}(z) & =\left(\frac{1}{2^{n} n ! \sqrt{\pi} l_{z}}\right)^{1 / 2} \exp \left(-\frac{z^{2}}{2 l_{z}^{2}}\right) H_{n}\left(\frac{z}{l_{z}}\right), \\
\varepsilon_{n} & =\left(n+\frac{1}{2}\right) \hbar \omega_{z}, \quad n=0,1,2, \ldots
\end{aligned}
$$

where $H_{n}(x)$ is the $n^{t h}$ Hermite polynomial and $l_{z}$ is $\sqrt{\hbar / m^{\star} \omega_{z}}$. For the calculation of this paper, we need the following matrix elements $[6,7,17]$

$$
\begin{gathered}
\left\langle\xi|x| \xi^{\prime}\right\rangle=x_{0} \delta_{N, N^{\prime}} \delta_{k, k^{\prime}}+\left(l^{\prime} / \omega_{c}\right)\left[(N+1)^{1 / 2} \delta_{N^{\prime}, N+1}-N^{1 / 2} \delta_{N^{\prime}, N-1}\right] \delta_{k k^{\prime}} \\
\left|\left\langle\xi\left|e^{ \pm i \vec{q} r}\right| \xi^{\prime}\right\rangle\right|^{2}=\left|I_{n, n^{\prime}}\left(q_{z}\right)\right|^{2}\left|J_{N, N^{\prime}}(u)\right|^{2} \delta_{k_{y}, k_{y}^{\prime} \pm q_{y}} \\
\left|J_{N, N^{\prime}}(u)\right|^{2}=\left(N^{\prime} ! / N !\right) e^{-u} u^{N^{\prime}-N}\left[L_{N}^{N^{\prime}-N^{\prime}}\right]^{2} \\
I_{n, n^{\prime}}\left( \pm q_{z}\right)=\frac{1}{\sqrt{2^{n} n ! 2^{n^{\prime}} n^{\prime} !} \sqrt{\pi} l_{z}} \int_{-\infty}^{\infty} e^{ \pm i q_{z} z} H_{n}\left(\frac{z}{l_{z}}\right) H_{n^{\prime}}\left(\frac{z}{l_{z}}\right) d z
\end{gathered}
$$

where $l^{\prime}=(l / \sqrt{2}) \omega_{c}, \delta_{k k^{\prime}}=\delta_{k_{y} k_{y}^{\prime}} \delta_{n n^{\prime}}, u=l^{2}\left(q_{x}^{2}+q_{y}^{2}\right) / 2=l^{2} q_{\perp}^{2} / 2$, and where $L_{N}^{M}(u)$ is an associated Laguerre polynomial.

For the $\sigma_{x x}^{d}$ components of magnetoconductivity, from (2) we have

$$
\sigma_{x x} \equiv \sigma_{x x}^{d}(0)=\frac{\beta e^{2}}{\Omega} \sum_{\xi, \xi^{\prime}}<n_{\xi}>_{e q}\left(1-<n_{\xi^{\prime}}>_{e q}\right) w_{\xi \xi^{\prime}}\left(X_{\xi}-X_{\xi^{\prime}}\right)^{2},
$$

where we have denoted $X_{\xi} \equiv R_{x \xi}=\langle\xi|x| \xi\rangle$. Inserting (3) and Eqs. (11-14) into (15), we derive the following form for magnetoconductivity

$$
\begin{array}{r}
\sigma_{x x}=\frac{\beta e^{2} A_{0}}{2 \pi^{3} \hbar l_{B}^{2}} \sum_{N, N^{\prime}, n, n^{\prime}} f_{N, n}\left(1-f_{N^{\prime}, n^{\prime}}\right) I\left(n, n^{\prime}\right) \int_{0}^{+\infty}|C(q)|^{2}\left|J_{N N^{\prime}}(u)\right|^{2} u d u \\
\times\left[N_{0} \delta\left(-P \hbar \omega_{c}+\left(n-n^{\prime}\right) \hbar \omega_{z}+\hbar \omega_{\vec{q}}\right)+\left(1+N_{0}\right) \delta\left(P \hbar \omega_{c}+\left(n-n^{\prime}\right) \hbar \omega_{z}-\hbar \omega_{\vec{q}}\right)\right],
\end{array}
$$

where we have set $I\left(n, n^{\prime}\right) \equiv \int_{-\infty}^{+\infty}\left|I_{n, n^{\prime}}\left( \pm q_{z}\right)\right|^{2} d q_{z},\left\langle n_{\xi}\right\rangle_{e q} \equiv f_{N, n}$, and $A_{0}$ is the surface area. To derive (16), we have transformed the sum over $\vec{q}$ into integral form in the usual way using cylindrical coordinates, and set $N^{\prime}-N=-P$ in the emission term and $N^{\prime}-N=P$ in the absorption term. Expression (16) for magnetoconductivity is general and can be applied for various electron-phonon scattering mechanism. We now assume the case where only intraelectric subband transitions $\left(n \rightarrow n^{\prime}=n\right)$ are allowed to take place and consider two kinds of phonons. 


\section{III.1. Nonpolar Optical Phonon Scattering}

For nonpolar-LO-phonon scattering, we take $\hbar \omega_{\vec{q}} \simeq \hbar \omega_{L} \simeq$ const $\left(\omega_{L}\right.$ is the LOphonon frequency), and $|C(q)|^{2}=\hbar D^{2} / 2 \Omega \rho \omega_{L}=D^{\prime} / \Omega$, where $\rho$ is the density of material and $D^{\prime}$ is the constant of the nonpolar interaction. Also, for nondegenerate semiconductors, the distribution function of electrons can be replaced by the Boltzmann distribution, i. e., $f_{N, n}=\exp \left[\beta\left(\varepsilon_{F}-\varepsilon_{N, n, k_{y}}\right)\right], \varepsilon_{F}$ is the Fermi level. From (16), after performing the integral over $u$ and the simple calculation, we obtain the following expression for magnetoconductivity

$$
\begin{aligned}
\sigma_{x x}= & \frac{\beta e^{2} D^{\prime}}{2 \pi^{3} l_{B}^{2} \hbar^{2} \omega_{c} l_{z}} \sum_{P, N, n} I(n, n) \exp \left[-\beta\left(\varepsilon_{N, n, k_{y}}-\varepsilon_{F}\right)\right] \\
& \times\left[(2 N+1)\left(2 N_{0}+1\right)-P\right] \delta\left(P-\frac{\omega_{L}}{\omega_{c}}\right) .
\end{aligned}
$$

\section{III.2. Polar Optical Phonon Scattering}

The only difference from above section is that $|C(q)|^{2}=A / \Omega q^{2}=A / \Omega\left(q_{\perp}^{2}+q_{z}^{2}\right) \approx$ $A l_{B}^{2} / 2 \Omega u$, where $A$ is a constant of the polar interaction. We assumed that $q_{\perp} \gg q_{z}$ for transport in the $(x, y)$ plane. This approximation allows us to do separately the integrals over $q_{z}$ and $u$ in (16). Proceeding with (16) as in the case of nonpolar optical phonon scattering, we have

$$
\sigma_{x x}=\frac{\beta e^{2} A}{4 \pi^{3} \hbar^{2} \omega_{c} l_{z}} \sum_{P, N, n} I(n, n) \exp \left[-\beta\left(\varepsilon_{N, n, k_{y}}-\varepsilon_{F}\right)\right]\left(2 N_{0}+1\right) \delta\left(P-\frac{\omega_{L}}{\omega_{c}}\right) .
$$

Equations (17) and (18) show $\delta$-function singularities of conductivity at resonance, $P \omega_{c}=\omega_{L}$. The sum over $P$ can be performed with the help of Poisson's summation formula as follow $[6,7,16]$

$$
\sum_{P=0}^{\infty} \delta\left(P-\omega_{L} / \omega_{c}\right) \approx 1+2 \sum_{s=1}^{\infty} e^{-2 \pi s\left(\Gamma_{N} / \hbar \omega_{c}\right)} \cos \left[2 \pi s\left(\omega_{L} / \omega_{c}\right)\right]
$$

where $\Gamma_{N}$ is the damping factor associated with the energy relaxation time $\tau$ by $\Gamma_{N} \approx \hbar / \tau$, $1 / \tau=\sum_{\xi^{\prime}} w_{\xi \xi^{\prime}}=\sum_{N^{\prime}, n^{\prime}, k_{y}^{\prime}} w_{N n k_{y} N^{\prime} n^{\prime} k_{y}^{\prime}}$. The equation for $\Gamma_{N}$ has a complicated form and will be solved numerically with the help of computer program. The numerical results are shown in the next section.

\section{NUMERICAL RESULTS AND DISCUSSION}

In order to clarify the mechanism of magnetophonon resonance effect in parabolic quantum wells, in this section, we numerically evaluate, plot the component $\sigma_{x x}$ of magnetoconductivity tensors for a specific parabolic quantum well. The characteristic parameters of sample are: $\varepsilon_{F}=50 \mathrm{meV}, \hbar \omega_{L}=36.25 \mathrm{meV}, \rho=5320 \mathrm{kgm}^{-3}, m=0.067 m_{0}\left(m_{0}\right.$ is mass of free electron).

Figures 1 and 2 show the dependence of magnetoconductivity $\sigma_{x x}$ on magnetic field $B$ in cases of nonpolar optical phonon scattering and polar optical phonon scattering, respectively, at $T=300 \mathrm{~K}$. For both cases, we can see that each curve has two maximum 


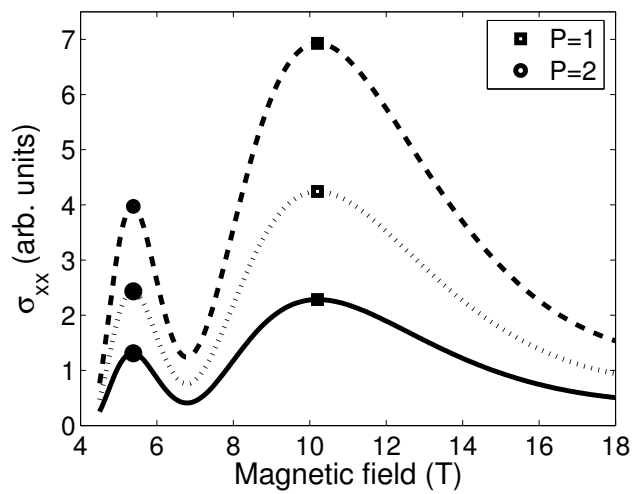

Fig. 1. The dependence of $\sigma_{x x}$ on $B$ at three different values of $\omega_{z}$ (nonpolar optical phonon scattering): $\omega_{z}=0.1 \times \omega_{L}$ (solid line), $\omega_{z}=$ $1.0 \times \omega_{L}$ (dashed line), and $\omega_{z}=2.0 \times \omega_{L}$ (dotted line).

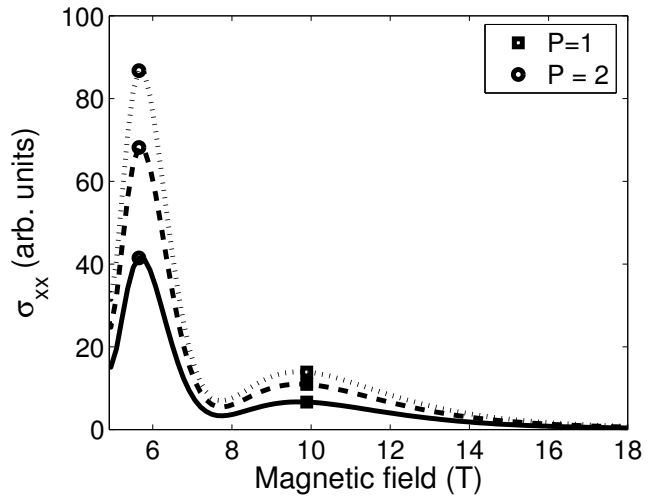

Fig. 2. The dependence of $\sigma_{x x}$ on $B$ at three different values of $\omega_{z}$ (polar optical phonon scattering): $\omega_{z}=0.3 \times \omega_{L}$ (solid line), $\omega_{z}=$ $0.5 \times \omega_{L}$ (dashed line), and $\omega_{z}=0.7 \times \omega_{L}$ (dotted line).

peaks. These maxima appear at the values of magnetic $B$ satisfying the resonant condition $P \omega_{c}=\omega_{L}$. If we take $\hbar \omega_{L}=36.25 \mathrm{meV}$, from the relation $\omega_{c}=e B / m^{\star}$, it is very easy to conclude that only $P=1$ and $P=2$ satisfy this condition. The fairly difference here is for the case of nonpolar optical phonon, the value of $\sigma_{x x}$ at $P=1$ is smaller than it is at $P=2$. This is contrast with the case of polar optical phonon. In addition, the values of $P$ are remained at different values of $\omega_{z}$. This is, of course, because of the absence of $\omega_{z}$ in

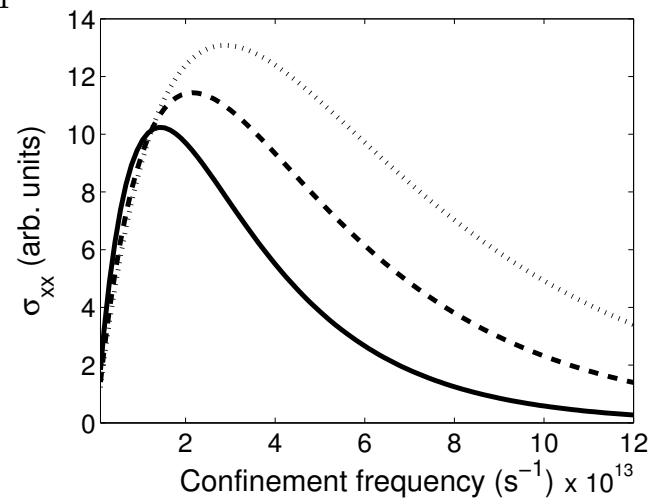

Fig. 3. The dependence of $\sigma_{x x}$ on $\omega_{z}$ at three different values of $T$ (nonpolar optical phonon scattering): $T=100 \mathrm{~K}$ (solid line), $T=150$ $\mathrm{K}$ (dashed line), and $T=200 \mathrm{~K}$ (dotted line). $B=10 \mathrm{~T}$.

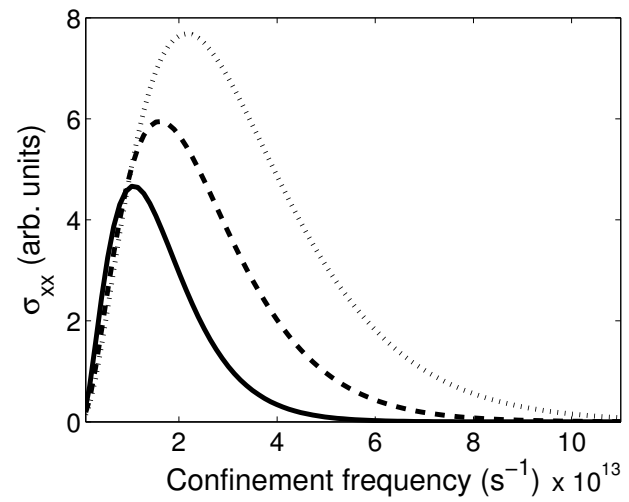

Fig. 4. The dependence of $\sigma_{x x}$ on $\omega_{z}$ at three different values of $T$ (polar optical phonon scattering): $T=100 \mathrm{~K}$ (solid line), $T=150$ $\mathrm{K}$ (dashed line), and $T=200 \mathrm{~K}$ (dotted line). $B=10 \mathrm{~T}$.

In figures 3 and 4 , we plot the magnetoconductivity versus the confinement frequency associated with nonpolar optical phonon and polar optical phonon scattering at 
three different values of temperature. It can be seen that every curve has a maximum value. When the temperature $T$ increases, this maxima shifts to the region corresponding to the larger values of $\omega_{z}$. Also, the larger the value of temperature is, the larger the value of magnetoconductivity is. For both cases, $\sigma_{x x}$ decreases when the confinement frequency increases. In case of polar optical phonon (Fig. 4), however, it diminishes more rapidly than it does in case of nonpolar optical phonon (Fig. 3).

\section{CONCLUSION}

In this paper, we have studied the MPR effect in a parabolic quantum well characterized by the confinement potential, assumed to be in $z$ direction, in the presence of a magnetic field $\vec{B}=B \vec{e}_{z}$. By using projection operators method, we have calculated the component $\sigma_{x x}$ of magnetoconductivity tensor, as well as the damping factor $\Gamma_{N}$, in the case of electron-optical phonon scattering with two types of optical phonon: nonpolar optical phonon and polar optical phonon. For both nonpolar- and polar-optical phonon scattering show the same resonant behaviors at $P \omega_{c}=\omega_{L}$ ( $P$ is an integer). If we take $\hbar \omega_{L}=36.25 \mathrm{meV}$, the possible values for $P$, that satisfy the resonant conditions, are 1 and 2. Furthermore, the dependence of $\sigma_{x x}$ on the confinement frequency is shown clearly for both cases of scattering mechanisms.

Finally, we notice that, with regard to the results for $\sigma_{x x}$, the usual divergences of the Born approximation have been avoided by replacing, as usual, the $\delta$ function by Lorentzians (or Gaussians). The relevant damping factors have been estimated from the inverse scattering rates but they have complicated forms and have been numerically evaluated in this work, with the help of computer, whereas they have been previously treated only at the resonances as in references [6]- [12].

\section{REFERENCES}

[1] R. J. Nicholas, Prog. Quant. Electr. 10 (1985) 1

[2] P. G. Harper, J. W. Hodby and R. A. Stradling, Rep. Progr. Phys. 37 (1973) 1

[3] B. K. Ridley, Quantum Processes in Semiconductor (Claredon Press, Oxford) (1993) 316

[4] A. Suzuki, Physica B, 183 (1998) 191

[5] S. C. Lee, Y. B. Kang, J. Y. Ryu, G. Y. Hu and S. D. Choi, Phys. Rev. B 57 (1998) 11875

[6] P. Vasilopoulos, Phys. Rev. B, 33 (1986) 8587

[7] P. Vasilopoulos, M. Charbonneau and C. M. van Vliet, Phys. Rev. B 35 (1987) 1334

[8] Sang Chil Lee, J. Korean Phys. Soc., 51 (2007) 1979

[9] P. Warmenbol, F. M. Peeters and J. T. Devreese, Phys. Rev. B 39 (1989) 7821; 37 (1988) 4694

[10] N. Mori. H. Murata, K. Taniguchi and C. Hamaguchi, Phys. Rev. B 38 (1988) 7622

[11] J. R. Barker, J. Phys. C 5 (1972) 1657; Solid State Electron., 21 (1978) 197

[12] J. Y. Ryu, B. Y. Kang, S. Oh, A. Suzuki and S. D. Choi, Phys. Rev. B 52 (1995) 11089

[13] N. Mori, H. Momose and C. Hamaguchi, Phys. Rev. B 45 (1992) 4536

[14] G. Q. Hai and F. M. Peeters, Phys. Rev. B 60 (1999) 16513

[15] S. C. Lee, J. Y. Ryu, S. W. Kim and C. S. Ting, Phys. Rev. B 62 (2000) 5045

[16] K. M. van Vliet, J. Math. Phys. 20 (1978) 2573

[17] M. Charbonneau, K. M. van Vliet, and P. Vasilopoulos, J. Math. Phys. 23 (1982) 318

[18] P. N. Argyres and L. M. Roth, J. Phys. Chem. Solids 12 (1959) 89

Received 30 September 2009. 